\title{
ALLELOPATHIC ACTIVITY OF CROP RESIDUE INCORPORATION ALONE OR MIXED AGAINST RICE AND ITS ASSOCIATED GRASS WEED JUNGLE RICE (Echinochloa colona $[$ L.] Link)
}

\author{
Abdul Khaliq ${ }^{1 *}$, Amar Matloob ${ }^{1}$, Zahid Ata Cheema ${ }^{1}$, and Muhammad Farooq ${ }^{1}$
}

Weed suppression is one of the several benefits achieved by soil incorporation of crop residues and such suppression is believed to be allelopathic in nature. The allelopathic potential of different crop residues: viz. sorghum (Sorghum bicolor [L.] Moench), sunflower (Helianthus annuus L.), brassica (Brassica campestris L.) was evaluated in rice (Oryza sativa L.) and jungle rice (Echinochloa colona [L.] Link). Chopped crop residues were soil-incorporated alone and mixed at 6 $\mathrm{g} \mathrm{kg}^{-1}$ soil $\left(12 \mathrm{t} \mathrm{ha}^{-1}\right)$ and compared with a control without residues. Soil incorporation of residues substantially delayed germination of jungle rice. The time to start germination, time to $50 \%$ emergence, mean emergence time, emergence index, and final germination percentage were all depressed by residue incorporation. Final germination of rice and jungle rice dropped by 11 to $15 \%$ and 11 to $27 \%$ with residue application alone and by 18 to $22 \%$ and 8 to $34 \%$ with a combination of crop residues, respectively. Residues were more suppressive to germination dynamics of jungle rice than rice. Crop residues exerted a pronounced negative influence on the shoot (25 to $100 \%$ and 14 to $44 \%$ ) and root lengths (22 to $100 \%$ and 10 to $43 \%$ ) of rice and jungle rice, respectively. Shoot and root dry weight of both rice and jungle rice also decreased significantly. An appreciable quantity of phenolics was recorded in soil amended with sorghum+sunflower+brassica residues. Since soil incorporation of allelopathic crop residues was detrimental to both rice germination and seedling growth, it is suggested that the time of residue application for jungle rice suppression and rice seeding time need to be adjusted so as to minimize rice crop damage.

Key words: Residues, allelopathy, sorghum, sunflower, brassica, Oryza sativa, Echinochloa colona, weed control.

$\mathrm{M}$ odern agriculture is productivity-oriented and relies primarily on synthetic inputs to tackle weeds and other pest problems (Sadeghi et al., 2010). Intensive herbicide use to control weeds over the last few decades is posing serious ecological and environmental threats to the planet and its inhabitants. Herbicide residues in produce, soil and ground water, shifts in weed populations, evolution of resistant weed biotypes, and associated health hazards have diverted the attention of researchers to discover and establish alternative weed management strategies. There is an increasing thrust for organicallyproduced commodities worldwide (Jamil et al., 2009). Allelopathy, an important ecological phenomenon that explains interference among species through biochemical pathways is a tool that can be manipulated to manage weeds in agroecosystems (Khanh et al., 2005). Use of allelopathic properties of native plant/crop species offers promising opportunities for this purpose. Allelopathy can regulate plant biodiversity through its impact on plant adaptation, survival, and community organization (Chou and Lee, 1991).

${ }^{1}$ University of Agriculture, Department of Agronomy, Faisalabad, Pakistan. "Corresponding author (khaliquaf@gmail.com).

Received: 24 December 2010.

Accepted: 15 April 2011.
Soil incorporation or surface application, such as mulch of allelopathic crop residues, affects weed dynamics by reducing/delaying seed germination and establishment, in addition to suppressing individual plant growth resulting in an overall decline in the density and vigor of the weed community (Gallandt et al., 1999). Allelopathic crop residue decomposition produces a variety of phytotoxins in the soil causing adverse effects on other plants (Nelson, 1996), and have the potential to sustain a chemical as well as physical effect on the growth and development of subsequent crops and weeds (Reddy, 2001). Allelopathic crop residues can be exploited for weed suppression, and can thus be helpful in reducing reliance on herbicides (Weston, 1996).

Sorghum (Sorghum bicolor [L.] Moench), sunflower (Helianthus annuus L.), and brassica (Brassica compestris L.) are potent allelopathic crops grown in ricebased cropping systems in South Asia, in general, and more particularly in the Indo-Pak region. Phytotoxicity of dried sunflower residues and leaf powder has been reported (Batish et al., 2002). Incorporation (in situ) of whole sorghum plant or its various parts, alone or mixed, was found to suppress weed growth in wheat (Triticum aestivum L.) (Cheema and Khaliq, 2000). Brassica campestris exhibited an inhibitory effect on weed density in the following year due to its residue decomposition 
(Norsworthy et al., 2005). Boydston and Hang (1995) found that incorporatiing Brassica napus residue reduced weed count and biomass by 73 to $85 \%$ and 50 to $96 \%$ in a potato (Solanum tuberosum L.) field.

Jungle rice (Echinochloa colona [L.] Link) is found to be a predominant weed in transplanted and direct-seeded rice (Rao et al., 2007), but is more competitive in the latter (Dubey, 2004). Besides rice, it also infests maize fields. It is the major grass weed with an intensified seed bank encountered early in the season in dry-seeded rice in Pakistan and is highly competitive (Caton et al., 2004).

Different plant species contain allelochemicals (phenolics being most abundant) that vary in type and concentration (Xuan et al., 2004). Weeds can be better controlled by incorporating plant residues that release a greater fraction of allelochemicals in the soil (Elijarrat and Barcelo, 2001). Release of allelochemicals by mixed residues can have a synergistic and/or additive effect on target species (Matloob et al., 2010). Although useful levels of herbicidal activity through residue incorporation have been achieved, there are still undesirable instances of phytotoxicity to subsequent crops (Holmes and Mayberry, 1996; Mitchell et al., 2000). As a practical approach, residue-mediated phytotoxins must selectively target weed species and not crop plants (Kruidohf et al., 2010). The present studies were carried out to investigate the possible suppressive effects of allelopathic sorghum, sunflower, and brassica residues against the germination dynamics and seedling growth of rice, and jungle rice, its associated grass weed. Moreover, phenolic release by the incorporated residues was quantified over time intervals and related to their suppressive activity.

\section{MATERIALS AND METHODS}

\section{Plant residues}

Field-grown mature sorghum ('JS-263'), sunflower ('Hysun-33'), and brassica ('Rainbow') plants were collected from the Agronomic Research Area, University of Agriculture, Faisalabad. Plants were chopped into 3 to $5-\mathrm{cm}$ pieces with a fodder cutter and oven-dried at $70{ }^{\circ} \mathrm{C}$ for $48 \mathrm{~h}$. Whole plant residues were mixed in situ into the soil.

\section{Bioassay}

Rice and jungle rice seeds were cleaned manually to ensure physical purity. These were surface sterilized with a water:bleach solution (10:1) for $15 \mathrm{~min}$ and rinsed four times with distilled water. Plastic pots $(29 \times 18 \mathrm{~cm}, 10$ $\mathrm{kg}$ capacity) were filled with air-dried, sieved, and wellmixed soil taken from the Agronomic Research Area. Soil belongs to the Lyallpur soil series (Aridisol-finesilty, mixed, hyperthermic Ustalfic, Haplargid) in the USDA classification and Haplic Yermosols in the FAO classification (Cheema and Khaliq, 2000). The $\mathrm{pH}$ of the saturated soil paste was 7.6 and total soluble salts were 1.2 $\mathrm{dS} \mathrm{m}^{-1}$. Organic matter, total $\mathrm{N}$, available $\mathrm{P}$ and $\mathrm{K}$ were
$0.71 \%, 0.062 \%, 13.1 \mathrm{mg} \mathrm{kg}^{-1}$, and $179 \mathrm{mg} \mathrm{kg}^{-1}$, respectively. Crop residues (sorghum, sunflower, and brassica) alone and combined in equal ratios were incorporated to obtain a total of $6 \mathrm{~g} \mathrm{~kg}^{-1}$ of soil $\left(12 \mathrm{t} \mathrm{ha}^{-1}\right)$. Pots without residue were included as a control. Two days after residue incorporation, 30 rice and jungle rice seeds were sown in each pot and these were placed in a screen house under natural solar radiation with an average temperature of $35 \pm 5^{\circ} \mathrm{C}$ and irrigated when required to keep the soil moist and avoid water stress. The experiment was repeated four times and visited daily to record emergence count (AOSA, 1990) until a constant count was achieved. The time lapse to 50\% emergence $\left(\mathrm{E}_{50}\right)$ was computed by following the modified formulae of Farooq et al. (2005):

$$
E_{50}=t_{i}+\frac{\left(\frac{N}{2}-n_{i}\right)\left(n_{j}-n_{i}\right)}{n_{j}-n_{i}}
$$

where $\mathrm{N}$ is the final number of emerged seeds, $n_{i}$ and $n_{j}$ are the cumulative number of seeds emerged by adjacent counts at times $\mathrm{t}_{\mathrm{i}}$ and $\mathrm{t}_{\mathrm{j}}$, and $\mathrm{n}_{\mathrm{i}}<\mathrm{N} / 2<\mathrm{n}_{\mathrm{j}}$. Mean emergence time (MET) was calculated according to Ellis and Roberts (1981) as:

$$
\mathrm{MET}=\frac{\sum \mathrm{Dn}}{\sum \mathrm{n}}
$$

where $\mathrm{n}$ is the number of seeds emerged on day $\mathrm{D}$, and $\mathrm{D}$ is the number of days counted from the beginning of sprouting. The emergence index was calculated as described by AOSA (1983):

$$
\mathrm{EI}=\frac{\mathrm{N}^{\mathrm{o}} \text { of emerged seeds }}{\text { Days of first count }}+------+\frac{\mathrm{N}^{\circ} \text { of emerged seeds }}{\text { Days of final count }}
$$

Root and shoot lengths were measured after $28 \mathrm{~d}$. Plants were uprooted after wetting with water, washed under the tap, and separated into roots and shoots from each pot. The number of leaves and secondary roots were counted manually and averaged. Harvested plant material was oven-dried at $70{ }^{\circ} \mathrm{C}$ for $48 \mathrm{~h}$ and dry root and shoot biomass was recorded. Total seedling biomass was taken as the sum of root and shoot biomass. Seedling mortality was calculated as:

$$
\text { Seedling mortality }(\%)=\frac{\begin{array}{c}
\mathrm{N}^{\circ} \text { of seedlings } \\
\text { emerged }-\mathrm{N}^{\circ} \text { of } \\
\text { seedling survived }
\end{array}}{\begin{array}{c}
\mathrm{N}^{\circ} \text { of seedlings } \\
\text { emerged }
\end{array}} \times 100
$$

Total water-soluble phenolic content of residueamended soil was determined at $700 \mathrm{~nm}$ wavelength in a UV-spectrophotometer (UV-4000, ORI, Hamburg, Germany) according to Swain and Hillis (1959) with Folin-Ciocalteu reagent and expressed as ferulic acid equivalents.

\section{Experimental design and statistical analysis}

Experimental pots were arranged in a completely randomized design with three replicates. Two separate 
experiments were conducted. Data were analyzed by ANOVA with MSTAT-C software (Freed and Scott, 1986). Treatment means were separated by the least significant differences (LSD) at $\mathrm{P} \leq 0.05$.

\section{RESULTS AND DISCUSSION}

\section{Germination traits}

Germination traits of rice and jungle rice were negatively influenced by crop residue incorporation (Table 1). Significant $(\mathrm{P} \leq 0.05)$ delay in the time to start germination and $\mathrm{E}_{50}$ over the control was provoked by all the treatments on jungle rice. Germination was delayed by $>1 \mathrm{~d}$ in jungle rice while it remained unaffected for rice. The time to start germination of jungle rice was delayed to the maximum by the combined application of sunflower+brassica residues and was at par with the incorporation of only sunflower residues, sorghum+brassica residues, or a combination of all three residues (sorghum+sunflower+brassica). Combinations of crop residue incorporation exerted a marked negative influence on time needed for $50 \%$ emergence (12 to 34 and 12 to $21 \%$ ) than when applied alone ( 1 to 11 and 11 to $14 \%$ ) for rice and jungle rice, respectively. Final rice germination was suppressed by 11 to $27 \%$. Sunflower residues incorporated alone scored $15 \%$ inhibition while combined with sorghum accounted for $27 \%$ suppression. Final germination of jungle rice dropped from 8 to $34 \%$. Interestingly, sorghum+sunflower residues were the least effective in retarding jungle rice germination $(-8 \%)$; maximum inhibition was noticed with all three crop residues, which was at par with that achieved by incorporating only sunflower and brassica residues (22 and 21\%). Incorporation of only sorghum, sunflower, and brassica provided statistically similar suppression of final germination in both rice and jungle rice. In various combinations, sorghum+sunflower scored $33 \%$ followed by $25 \%$ inhibition recorded when all three residues were combined. Mean germination time (MGT) also reflected the allelopathic influence of crop residues on germination events and a significant increase over the control was recorded for both test species. A combination of sorghum+sunflower scored maximum (84\%) delay in MGT for rice while a similar suppression value for jungle rice was attributed to mixing all three crop residues. Significantly $(\mathrm{P} \leq 0.05)$ lower emergence index (EI) values, except for the control, were also noticed with minimum EI observed when all three residues were incorporated integratively.

A critical look into the data obtained confirmed the inhibitory effects of crop residues on germination and seedling growth of the test species. Such suppressive actions are believed to originate through the release of phytotoxins (allelochemicals) from incorporated crop residues either by leaching or decomposition (Birkett et al., 2001). Allelopathic compounds in crop residues were rapidly solubilized and imbibed by the germinating seeds, retarded/delayed emergence, and adversely affected subsequent seedling growth. Germination suppression of test species influenced by allelopathic crop residues is in line with Matloob et al. (2010).

\section{Seedling growth}

Rice and jungle rice root and shoot length were also significantly $(\mathrm{P} \leq 0.05)$ suppressed by crop residues and various combinations thereof (Table 2). These residues were significantly reduced in the root (22 to 100and 10 to $43 \%$ ) and shoot (25 to 100 and 14 to $44 \%$ ) length over the control in rice and jungle rice, respectively. A combination of sorghum+sunflower+brassica recorded

Table 1. Influence of crop residues applied alone or combined on rice and jungle rice germination traits.

\begin{tabular}{|c|c|c|c|c|c|c|c|c|c|c|}
\hline \multirow[b]{2}{*}{ Treatments } & \multicolumn{2}{|c|}{$\begin{array}{l}\text { Time to start } \\
\text { germination }(\mathrm{d})\end{array}$} & \multicolumn{2}{|c|}{$\begin{array}{c}\text { Final } \\
\text { germination }(\%)\end{array}$} & \multicolumn{2}{|c|}{$\begin{array}{l}\text { Time to } 50 \% \\
\text { emergence (d) }\end{array}$} & \multicolumn{2}{|c|}{$\begin{array}{l}\text { Mean germination } \\
\text { time }(d)\end{array}$} & \multicolumn{2}{|c|}{$\begin{array}{l}\text { Emergence } \\
\text { index }\end{array}$} \\
\hline & Rice & $\begin{array}{c}\text { Jungle } \\
\text { rice }\end{array}$ & Rice & $\begin{array}{l}\text { Jungle } \\
\text { rice }\end{array}$ & Rice & $\begin{array}{c}\text { Jungle } \\
\text { rice }\end{array}$ & Rice & $\begin{array}{l}\text { Jungle } \\
\text { rice }\end{array}$ & Rice & $\begin{array}{l}\text { Jungle } \\
\text { rice }\end{array}$ \\
\hline $\mathrm{T}_{1}$ Control & $4.00 \mathrm{~ns}$ & $8.00 \mathrm{c}$ & $86.67 \mathrm{a}$ & $78.89 a$ & $4.96 \mathrm{~d}$ & $10.55 \mathrm{c}$ & $5.54 \mathrm{~b}$ & $12.38 \mathrm{e}$ & $13.19 \mathrm{a}$ & $8.16 \mathrm{a}$ \\
\hline $\begin{array}{l}\mathrm{T}_{2} \text { Sorghum residues incorporated at } \\
6 \mathrm{~g} \mathrm{~kg}^{-1} \text { soil }\end{array}$ & $\begin{array}{l}4.00 \\
(0.00)\end{array}$ & $\begin{array}{l}8.33 b c \\
(4.17)\end{array}$ & $\begin{array}{l}74.44 b \\
(-14.11)\end{array}$ & $\begin{array}{l}64.44 \mathrm{bc} \\
(-18.31)\end{array}$ & $\begin{array}{l}5.02 \mathrm{~d} \\
(1.14)\end{array}$ & $\begin{array}{l}12.05 \mathrm{ab} \\
(14.22)\end{array}$ & $\begin{array}{l}5.77 b \\
(4.15)\end{array}$ & $\begin{array}{l}13.69 \mathrm{abcd} \\
(10.58)\end{array}$ & $\begin{array}{l}10.16 b c d \\
(-22.95)\end{array}$ & $\begin{array}{l}5.93 \mathrm{bc} \\
(-27.37)\end{array}$ \\
\hline $\begin{array}{l}\mathrm{T}_{3} \text { Sunflower residues incorporated at } \\
6 \mathrm{~g} \mathrm{~kg}^{-1} \text { soil }\end{array}$ & $\begin{array}{l}4.00 \\
(0.00)\end{array}$ & $\begin{array}{l}9.00 \mathrm{ab} \\
(12.50)\end{array}$ & $\begin{array}{l}73.33 \mathrm{ab} \\
(-15.39)\end{array}$ & $\begin{array}{l}61.11 \mathrm{~cd} \\
(-22.54)\end{array}$ & $\begin{array}{l}5.41 \mathrm{~cd} \\
(9.07)\end{array}$ & $\begin{array}{l}11.94 b \\
(13.18)\end{array}$ & $\begin{array}{l}6.87 \mathrm{~b} \\
(24.01)\end{array}$ & $\begin{array}{l}13.24 \mathrm{~cd} \\
(6.97)\end{array}$ & $\begin{array}{l}11.16 \mathrm{abc} \\
(-15.42)\end{array}$ & $\begin{array}{l}5.19 \mathrm{c} \\
(-36.32)\end{array}$ \\
\hline $\begin{array}{l}\mathrm{T}_{4} \text { Brassica residues incorporated at } \\
6 \mathrm{~g} \mathrm{~kg}^{-1} \text { soil }\end{array}$ & $\begin{array}{l}4.00 \\
(0.00)\end{array}$ & $\begin{array}{l}8.33 \mathrm{bc} \\
(4.17)\end{array}$ & $\begin{array}{l}76.67 \mathrm{ab} \\
(-11.54)\end{array}$ & $\begin{array}{l}62.22 \mathrm{bcd} \\
(-21.13)\end{array}$ & $\begin{array}{l}5.50 \mathrm{~cd} \\
(10.95)\end{array}$ & $\begin{array}{l}11.72 b \\
(11.09)\end{array}$ & $\begin{array}{l}6.90 \mathrm{~b} \\
(24.55)\end{array}$ & $\begin{array}{l}13.13 \mathrm{~d} \\
(6.06)\end{array}$ & $\begin{array}{l}11.54 \mathrm{ab} \\
(-12.48)\end{array}$ & $\begin{array}{l}5.92 \mathrm{bc} \\
(-27.49)\end{array}$ \\
\hline $\begin{array}{ll}\mathrm{T}_{5} & \text { Sorghum + sunflower residues } \\
\text { each incorporated at } 3 \mathrm{~g} \mathrm{~kg}^{-1} \text { soil }\end{array}$ & $\begin{array}{l}4.67 \\
(16.67)\end{array}$ & $\begin{array}{l}8.33 b c \\
(4.17)\end{array}$ & $\begin{array}{l}63.34 b \\
(-26.93)\end{array}$ & $\begin{array}{l}72.22 \mathrm{ab} \\
(-8.45)\end{array}$ & $\begin{array}{l}6.39 \mathrm{ab} \\
(28.97)\end{array}$ & $\begin{array}{l}11.84 b \\
(12.26)\end{array}$ & $\begin{array}{l}10.21 \mathrm{a} \\
(84.36)\end{array}$ & $\begin{array}{l}13.44 \mathrm{bcd} \\
(8.56)\end{array}$ & $\begin{array}{l}8.13 d \\
(-38.34)\end{array}$ & $\begin{array}{l}7.59 \mathrm{ab} \\
(-7.03)\end{array}$ \\
\hline $\begin{array}{l}\mathrm{T}_{6} \text { Sorghum + brassica residues each } \\
\text { incorporated at } 3 \mathrm{~g} \mathrm{~kg}^{-1} \text { soil }\end{array}$ & $\begin{array}{l}4.67 \\
(16.67)\end{array}$ & $\begin{array}{l}9.33 \mathrm{a} \\
(16.67)\end{array}$ & $\begin{array}{l}76.67 b \\
(-11.54)\end{array}$ & $\begin{array}{l}66.67 b c \\
(-15.49)\end{array}$ & $\begin{array}{l}5.57 \mathrm{~cd} \\
(12.30)\end{array}$ & $\begin{array}{l}11.95 b \\
(13.27)\end{array}$ & $\begin{array}{l}7.29 \mathrm{ab} \\
(31.53)\end{array}$ & $\begin{array}{l}13.81 \mathrm{abc} \\
(11.55)\end{array}$ & $\begin{array}{l}11.38 \mathrm{ab} \\
(-13.70)\end{array}$ & $\begin{array}{l}6.05 b c \\
(-25.82)\end{array}$ \\
\hline $\begin{array}{l}\mathrm{T}_{7} \text { Sunflower }+ \text { brassica residues each } \\
\text { incorporated at } 3 \mathrm{~g} \mathrm{~kg}^{-1} \text { soil }\end{array}$ & $\begin{array}{l}4.00 \\
(0.00)\end{array}$ & $\begin{array}{l}9.67 \mathrm{a} \\
(20.83)\end{array}$ & $\begin{array}{l}73.33 b \\
(-15.39)\end{array}$ & $\begin{array}{l}62.22 \mathrm{bcd} \\
(-21.13)\end{array}$ & $\begin{array}{l}5.81 \mathrm{bc} \\
(17.07)\end{array}$ & $\begin{array}{l}12.32 \mathrm{ab} \\
(16.75)\end{array}$ & $\begin{array}{l}7.47 \mathrm{ab} \\
(34.78)\end{array}$ & $\begin{array}{l}14.00 \mathrm{ab} \\
(13.09)\end{array}$ & $\begin{array}{l}11.54 \mathrm{ab} \\
(-12.48)\end{array}$ & $\begin{array}{l}5.87 \mathrm{bc} \\
(-28.02)\end{array}$ \\
\hline $\begin{array}{ll}\mathrm{T}_{8} & \text { Sorghum }+ \text { sunflower }+ \text { brassica } \\
\text { residues each incorporated at } 2 \mathrm{~g} \\
\mathrm{~kg}^{-1} \text { soil }\end{array}$ & $\begin{array}{l}4.67 \\
(16.67)\end{array}$ & $\begin{array}{l}9.67 \mathrm{a} \\
(20.83)\end{array}$ & $\begin{array}{l}71.11 b \\
(-17.95)\end{array}$ & $\begin{array}{l}52.22 \mathrm{~d} \\
(-33.80)\end{array}$ & $\begin{array}{l}6.68 \mathrm{a} \\
(34.68)\end{array}$ & $\begin{array}{l}12.79 \mathrm{a} \\
(21.23)\end{array}$ & $\begin{array}{l}7.31 \mathrm{ab} \\
(32.01)\end{array}$ & $\begin{array}{l}14.11 \mathrm{a} \\
(13.97)\end{array}$ & $\begin{array}{l}8.41 \mathrm{~cd} \\
(-36.26)\end{array}$ & $\begin{array}{l}4.47 \mathrm{c} \\
(-45.26)\end{array}$ \\
\hline $\operatorname{LSD}(\mathrm{P} \leq 0.05)$ & & 0.86 & 17.51 & 10.86 & 0.76 & 0.79 & 3.15 & 0.64 & 2.84 & 2.06 \\
\hline
\end{tabular}

"Means with different letters differ significantly at 5\% probability level; "*Figures shown in parenthesis show percent change over the control, ns: non significant.

LSD: least significant differences. 
Table 2. Influence of crop residues applied alone or combined on rice and jungle rice seedling growth.

\begin{tabular}{|c|c|c|c|c|c|c|c|c|c|c|c|c|}
\hline & \multicolumn{2}{|c|}{$\begin{array}{l}\text { Root length } \\
(\mathrm{cm})\end{array}$} & \multicolumn{2}{|c|}{$\begin{array}{l}\text { Shoot length } \\
(\mathrm{cm})\end{array}$} & \multicolumn{2}{|c|}{$\begin{array}{l}\text { Root dry weight } \\
\left(\mathrm{g} \text { seedling }^{-1}\right)\end{array}$} & \multicolumn{2}{|c|}{$\begin{array}{l}\text { Shoot dry weight } \\
\left(\mathrm{g} \text { seedling }^{-1}\right)\end{array}$} & \multicolumn{2}{|c|}{$\begin{array}{l}\text { Root } \\
\text { score }\end{array}$} & \multicolumn{2}{|c|}{$\begin{array}{c}\text { Mortality } \\
(\%)\end{array}$} \\
\hline & Rice & $\begin{array}{c}\text { Jungle } \\
\text { rice }\end{array}$ & Rice & $\begin{array}{c}\text { Jungle } \\
\text { rice }\end{array}$ & Rice & $\begin{array}{l}\text { Jungle } \\
\text { rice }\end{array}$ & Rice & $\begin{array}{c}\text { Jungle } \\
\text { rice }\end{array}$ & Rice & $\begin{array}{l}\text { Jungle } \\
\text { rice }\end{array}$ & Rice & $\begin{array}{c}\text { Jungle } \\
\text { rice }\end{array}$ \\
\hline $\mathrm{T}_{1}$ & $4.08 \mathrm{a}^{*}$ & $16.47 \mathrm{a}$ & $12.46 \mathrm{a}$ & $39.80 \mathrm{a}$ & $0.09 \mathrm{a}$ & $0.42 \mathrm{a}$ & $0.37 \mathrm{a}$ & $2.24 \mathrm{a}$ & $4.20 \mathrm{a}$ & $13.87 \mathrm{a}$ & - & - \\
\hline $\mathrm{T}_{2}$ & $\begin{array}{l}2.99 \mathrm{~cd} \\
(-26.80)^{* * *}\end{array}$ & $\begin{array}{l}13.60 b c \\
(-17.43)\end{array}$ & $\begin{array}{l}9.14 b \\
(-26.62)\end{array}$ & $\begin{array}{l}32.80 b \\
(-17.59)\end{array}$ & $\begin{array}{l}0.05 \mathrm{ab} \\
(-40.74)\end{array}$ & $\begin{array}{l}0.29 b c \\
(-31.27)\end{array}$ & $\begin{array}{l}0.23 b \\
(-38.74)\end{array}$ & $\begin{array}{l}1.46 b \\
(-34.73)\end{array}$ & $\begin{array}{l}1.72 \mathrm{bcd} \\
(-58.97)\end{array}$ & $\begin{array}{l}8.72 b c \\
(-37.11)\end{array}$ & $20.44 d$ & $23.14 \mathrm{ab}$ \\
\hline $\mathrm{T}_{3}$ & $\begin{array}{l}3.17 \mathrm{c} \\
(-22.39)\end{array}$ & $\begin{array}{l}11.47 d \\
(-30.38)\end{array}$ & $\begin{array}{l}7.94 b c \\
(-36.30)\end{array}$ & $\begin{array}{l}26.93 d \\
(-32.33)\end{array}$ & $\begin{array}{l}0.04 b \\
(-59.26)\end{array}$ & $\begin{array}{l}0.26 \mathrm{bcd} \\
(-38.41)\end{array}$ & $\begin{array}{l}0.16 b \\
(-55.86)\end{array}$ & $\begin{array}{l}1.37 b \\
(-38.87)\end{array}$ & $\begin{array}{l}2.24 b \\
(-46.59)\end{array}$ & $\begin{array}{l}7.41 \mathrm{~cd} \\
(-46.58)\end{array}$ & $17.75 \mathrm{e}$ & $14.85 \mathrm{~d}$ \\
\hline $\mathrm{T}_{4}$ & $\begin{array}{l}3.16 \mathrm{c} \\
(-22.47)\end{array}$ & $\begin{array}{l}12.87 \mathrm{~cd} \\
(-21.88)\end{array}$ & $\begin{array}{l}8.15 b c \\
(-34.59)\end{array}$ & $\begin{array}{l}34.13 b \\
(-14.24)\end{array}$ & $\begin{array}{l}0.04 b \\
(-59.26)\end{array}$ & $\begin{array}{l}0.28 b c d \\
(-34.76)\end{array}$ & $\begin{array}{l}0.17 b \\
(-53.15)\end{array}$ & $\begin{array}{l}1.44 b \\
(-35.57)\end{array}$ & $\begin{array}{l}1.50 \mathrm{~cd} \\
(-64.21)\end{array}$ & $\begin{array}{l}8.17 \mathrm{bcd} \\
(-41.10)\end{array}$ & $24.39 \mathrm{c}$ & $20.01 b c$ \\
\hline $\mathrm{T}_{5}$ & $\begin{array}{l}2.81 \mathrm{~d} \\
(-31.21)\end{array}$ & $\begin{array}{l}14.80 \mathrm{ab} \\
(-10.14)\end{array}$ & $\begin{array}{l}7.16 c \\
(-42.51)\end{array}$ & $\begin{array}{l}28.13 \mathrm{~cd} \\
(-29.31)\end{array}$ & $\begin{array}{l}0.02 \mathrm{~b} \\
(-74.07)\end{array}$ & $\begin{array}{l}0.31 b \\
(-24.76)\end{array}$ & $\begin{array}{l}0.13 b \\
(-63.96)\end{array}$ & $\begin{array}{l}1.51 b \\
(-32.62)\end{array}$ & $\begin{array}{l}1.47 \mathrm{~cd} \\
(-65.08)\end{array}$ & $\begin{array}{l}9.47 b \\
(-31.75)\end{array}$ & $24.53 b c$ & $17.87 \mathrm{~cd}$ \\
\hline $\mathrm{T}_{6}$ & $\begin{array}{l}3.74 b \\
(-8.42)\end{array}$ & $\begin{array}{l}12.87 \mathrm{~cd} \\
(-21.88)\end{array}$ & $\begin{array}{l}9.35 b \\
(-24.93)\end{array}$ & $\begin{array}{l}31.20 b c \\
(-21.61)\end{array}$ & $\begin{array}{l}0.03 b \\
(-62.96)\end{array}$ & $\begin{array}{l}0.21 \mathrm{de} \\
(-50.32)\end{array}$ & $\begin{array}{l}0.21 b \\
(-44.14)\end{array}$ & $\begin{array}{l}1.30 b \\
(-41.82)\end{array}$ & $\begin{array}{l}1.89 b c \\
(-55.08)\end{array}$ & $\begin{array}{l}8.22 b c d \\
(-40.74)\end{array}$ & $26.60 \mathrm{~b}$ & $18.52 \mathrm{~cd}$ \\
\hline $\mathrm{T}_{7}$ & $\begin{array}{l}2.60 \mathrm{e} \\
(-36.27)\end{array}$ & $\begin{array}{l}12.60 \mathrm{~cd} \\
(-23.50)\end{array}$ & $\begin{array}{l}7.56 b c \\
(-39.33)\end{array}$ & $\begin{array}{l}31.93 b \\
(-19.77)\end{array}$ & $\begin{array}{l}0.02 b \\
(-74.07)\end{array}$ & $\begin{array}{l}0.22 \mathrm{cde} \\
(-48.41)\end{array}$ & $\begin{array}{l}0.15 b \\
(-59.46)\end{array}$ & $\begin{array}{l}1.57 b \\
(-29.94)\end{array}$ & $\begin{array}{l}1.24 d \\
(-70.56)\end{array}$ & $\begin{array}{l}7.90 \mathrm{~cd} \\
(-43.02)\end{array}$ & $17.15 \mathrm{e}$ & $17.60 \mathrm{~cd}$ \\
\hline $\mathrm{T}_{8}$ & $\begin{array}{l}0.00 f \\
(-100.00)\end{array}$ & $\begin{array}{l}9.43 \mathrm{e} \\
(-42.72)\end{array}$ & $\begin{array}{l}0.00 \mathrm{~d} \\
(-100.00)\end{array}$ & $\begin{array}{l}22.27 \mathrm{e} \\
(-44.05)\end{array}$ & $\begin{array}{l}0.00 b \\
(-100.00)\end{array}$ & $\begin{array}{l}0.15 \mathrm{e} \\
(-63.17)\end{array}$ & $\begin{array}{l}0.00 c \\
(-100.00)\end{array}$ & $\begin{array}{l}0.90 c \\
(-59.58)\end{array}$ & $\begin{array}{l}0.00 \mathrm{e} \\
(-100.00)\end{array}$ & $\begin{array}{l}6.96 \mathrm{~d} \\
(-49.80)\end{array}$ & $100.00 \mathrm{a}$ & $26.67 \mathrm{a}$ \\
\hline LSD & 0.19 & 1.71 & 1.79 & 3.20 & 0.05 & 0.08 & 0.11 & 0.37 & 0.62 & 1.45 & 2.14 & 3.71 \\
\hline
\end{tabular}

"Means with different letters differ significantly at $5 \%$ probability level.

**Figures shown in parenthesis show percent change over the control.

LSD: least significant differences.

maximum suppression of these parameters in both test species. Shoot and root dry weight was also reduced under all treatments over the control. Among the application of residues alone, sunflower scored the maximum reduction in shoot (59 and 38\%) and root (56 and 39\%) dry weight of rice and jungle rice, respectively. A combination of all three residues proved to be even better in suppressing dry matter accumulation in test species. Residue incorporation led to reduced root proliferation as indicated by lower root scores compared to the control. Sunflower residues exerted a drastic influence on root score and suppressed it by $46 \%$ while its combination with brassica residues accounted for 70 and $43 \%$ inhibition in rice and jungle rice, respectively. Sorghum+sunflower+brassica residues caused $100 \%$ seedling mortality in rice while they killed up to $27 \%$ of jungle rice seedlings.

A number of allelochemicals responsible for inhibitory allelopathic activity at specific quantities have been reported for sorghum (Cheema et al., 2009), sunflower (Macias etal.,2002; Anjum and Bajwa, 2005), and brassica spp. (Al-Khatib and Boydston, 1999). Residue species varied in their severity against test species which also reflected variable susceptibility to different crop residue species and various combinations thereof. Such a variable influence of sorghum, sunflower, and brassica residues can be due to the type and concentration of allelochemicals involved. Allelopathic interactions are characterized by species specificity of both the donor and receiver (Rice, 1984), and manifested owing to the concerted action of numerous allelochemicals in the substratum (Einhellig, 1996). Blum (1996) reported that phenolic allelochemical mixtures and other organics present in the substratum could cause inhibitory effects even though the concentration of individual allelochemicals is below the required level to cause an effect. It is assumed that the increase in the magnitude of suppression with an increasing number of crop residues in the mixture in these studies was on account of the compound effect of the variety of allelochemicals (Matloob et al., 2010).

The variable influence of crop residues is also believed to be due to the differential seed size of the test species. Petersen et al. (2001) pointed out that small-seeded species are more subject to the adverse effects of phytotoxic crop residues. Several explanatory mechanisms and hypotheses have been proposed for this differential suppression. Firstly, small-seeded species have more root length per unit of root mass (Leishman et al., 2000) that provides a more absorbing surface area for allelochemical uptake. Secondly, bold-seeded species contain large seed reserves which are positively correlated with seed size. Thus, they enjoy a competitive edge over small-seeded species because of better seedling respiration under stress-induced carbon deficit conditions (Westoby et al., 2002). Liebman and Sundberg (2006) proposed that species with large reserves are also better able to tolerate and detoxify allelopathic agents. On the contrary, rice appeared more susceptible to phytotoxins than jungle rice in our study. Although it showed prompt germination, even under residue influence, seedling decay was observed with higher mortality rates. Shoot and root elongation, as well as dry matter accumulation in these parts, was relatively less than that recorded for jungle rice under the same set of treatments. This might be attributed to morphological and physiological divergence present in the test species. Moreover, given that weeds are more versatile, aggressive, and tough, they are better able to adapt to changing or adverse external conditions than crops.

\section{Phenolic dynamics}

Since phenolics are the major category of water-soluble allelochemicals responsible for most allelopathic activity, 
their presence was quantified over time after incorporation. An appreciable quantity of phenolics was encountered in soil amended with sorghum+sunflower+brassica residues (Figure 1). Phenolic content showed a periodic increase over time achieving peaks after $96 \mathrm{~h}$ after which a decrease in phenolic content was observed. Thus, releasing allelochemicals into the rhizosphere is a function of time, as well as quantity and type of residue involved. The decline in phenolic content of residueamended soil is due to the variety of physic-chemical and biological transformations when entering into the soil phase as proposed by Blum et al. (1999). This confirms the hypothesis by Kruidhof et al. (2010), who proposed that residue-mediated inhibition can occur only if the susceptibility period of the receptor plant coincides with the inhibitory allelopathic potential peak period. Our studies suggest that jungle rice was more susceptible to residue allelopathy during germination while rice sensitivity was attributable to seedling suppression. Khanh et al. (2005) affirmed that combining different crop plants can help to control more weed species due to its greater inhibitory potential, and that it must find weed initial growth in order to be effective.

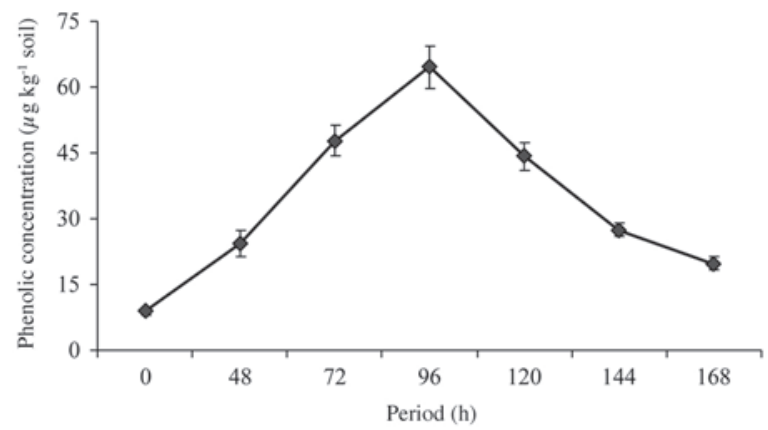

Figure 1. Dynamics of water soluble phenolics released in $2 \mathrm{~g} \mathrm{~kg}^{-1}$ soil amended with each of sorghum+sunflower+brassica residues.

\section{CONCLUSION}

These studies conclude that integrating sorghum, sunflower, and brassica residues has the potential to suppress jungle rice germination and seedling growth. These residues can be used as an eco-friendly approach to manage this weed, provided that maximum levels of phytotoxins entering into the soil find the early growth and development of this weed. Waiting 1 wk between residue incorporation and seeding a sensitive crop such as rice can increase crop safety and reduce the weed burden before seeding. However, field studies need to be conducted to evaluate the suppressive efficacy of such residues applied alone and combined with each other under natural conditions. Moreover, sorghum, sunflower, and brassica crop allelopathy can play an important role in different crop rotations to manage the spread of jungle rice.

\section{ACKNOWLEDGEMENTS}

The authors are grateful to the Higher Education Commission of Pakistan for providing financial support for these studies under the National Research Program.

Actividad alelopática de residuos de cultivo incorporados solos o en mezcla contra arroz y su maleza asociada pasto del arroz o pasto Shama (Echinochloa colona $[$ L.] Link). La represión de malezas es uno de los beneficios logrados por la incorporación de residuos de cosecha al suelo y se cree que es de naturaleza alelopática. Se evaluó el potencial alelopático de diversos residuos de cultivos: sorgo (Sorghum bicolor [L.] Moench), girasol (Helianthus annuus L.) y coles (Brassica Compestris L.) en arroz (Oryza sativa L.) y pasto arroz o pasto Shama (Echinochloa colona [L.] Link). Los residuos de cosecha picados se incorporaron solos y en mezcla a $6 \mathrm{~g} \mathrm{~kg}^{-1}$ de suelo $\left(12 \mathrm{t} \mathrm{ha}^{-1}\right)$ y se compararon con un testigo sin residuos. La incorporación de los residuos al suelo retrasó sustancialmente la germinación del pasto arroz o pasto Shama. El tiempo para empezar la germinación, el tiempo a $50 \%$ de emergencia, el tiempo medio de emergencia, el índice de emergencia, y el porcentaje de germinación final fueron influidos negativamente por la incorporación de residuos. La germinación final del arroz y del pasto arroz o pasto Shama se redujo en 11 a $15 \%$ y 11 a $27 \%$ con el uso exclusivo de residuos, y en 18 a $22 \%$ y 8 a $34 \%$ con la combinación de los residuos de cultivos, respectivamente. Los residuos de cultivos ejercieron una marcada influencia negativa en la longitud de brotes $(25-100 \%$ y $14-44 \%)$ y la longitud de raíces (22-100\% y $10-43 \%$ ) de arroz y de pasto arroz o pasto Shama, respectivamente. Peso seco de brotes y raíces de arroz y pasto del arroz o pasto Shama también decrecieron significativamente. Una apreciable cantidad de fenoles fue registrada en el suelo enmendado con residuos de sorgo+girasol+coles. Desde que los residuos alelopáticos de cultivos se incorporaron al suelo también fueron perjudiciales para la germinación y crecimiento de las plántulas de arroz, así se sugiere que el momento de la aplicación de los residuos para la supresión de arroz de la selva y el tiempo de la siembra del arroz deben ser ajustados para reducir al mínimo el daño del cultivo de arroz.

Palabras clave: residuos, alelopatía, sorgo, girasol, coles, Oryza sativa, Echinochloa colona, control de malezas.

\section{LITERATURE CITED}

Al-Khatib, K., and R. Boydston. 1999. Weed control with Brassica green manure crops. p. 255-270. In S.S. Narwal (ed.) Allelopathy update, basic and applied aspects. Oxford \& IBH Publisher, New Dehli, India.

Anjum, T., and R. Bajwa. 2005. A bioactive annuionone from sunflower leaves. Phytochemistry 66:1919-1921.

AOSA. 1983. Seed vigor testing handbook. Contribution $N^{\circ} 32$. Association of Official Seed Analysis (AOSA), Springfield, Illinois, USA. 
AOSA. 1990. Rules for testing seeds. Journal of Seed Technology 12:1-112.

Batish, D.R.,P.Tung, H.P.Singh, and R.K. Kohli. 2002.Phytotoxicity of sunflower residues against some summer season crops. Journal of Agronomy and Crop Science 188:19-24.

Birkett, M.A., K. Chamberlein, A.M. Hooper, and J.A. Pickett. 2001. Does allelopathy offer real promise for practical weed management and for explaining rhizosphere interactions involving higher plants. Plant and Soil 232:31-39.

Blum, U. 1996. Allelopathic interactions involving phenolic acids. Journal of Nematology 28:259-267.

Blum, U., S.R. Shafer, and M.E. Lehman. 1999. Evidence for inhibitory allelopathic interactions involving phenolic acids in field soils: concepts vs. experimental model. Critical Reviews in Plant Science 18:673-693.

Boydston, R.A., and A. Hang. 1995. Rape seed (Brassica napus) green manure crop suppresses weeds in potato (Solanum tuberosum). Weed Technology 9:669-675.

Caton, B.P., M. Mortimer, and J.H. Hill. 2004. A practical guide to weeds of rice in Asia. 58 p. International Rice Research Institute (IRRI), Los Baños, Laguna, Philippines.

Cheema, Z.A., and A. Khaliq. 2000. Use of sorghum allelopathic properties to control weeds in irrigated wheat in a semi arid region of Punjab. Agriculture Ecosystems \& Environment 79:105-112.

Cheema, Z.A., M.N. Mushtaq, M. Farooq, A. Hussain, and I.U. Din. 2009. Purple nutsedge management with allelopathic sorghum. Allelopathy Journal 23:305-312.

Chou, C.H., and Y.F. Lee. 1991. Allelopathic dominance of Miscanthus transnorrisonensis in an alpine grassland community in Taiwan. Journal of Chemical Ecology 17:2267-2281.

Dubey, V. 2004. Ecology of jungle rice (Echinochloa colonum), a weed of rice agroecosystems: a case study in Bilaspur (Chhattishgarh). International Rice Research Notes 29(2):52-55.

Einhellig, F.A. 1996. Interactions involving allelopathy in cropping systems: Allelopathy in cropping systems. Agronomy Journal 88:883-893

Elijarrat, E., and D. Barcelo. 2001. Sample handling and analysis of allelochemical compounds in plants. Trends in Analytical Chemistry 20:584-590.

Ellis, R.A., and E.H. Roberts. 1981. The quantification of ageing and survival in orthodox seeds. Seed Science \& Technology 9:373409.

Farooq, M., S.M.A. Basra, K. Hafeez, and N. Ahmad. 2005. Thermal hardening: a new seed vigor enhancing tool in rice. Journal of Integrative Plant Biology 47:187-193.

Freed, R.D., and D.E. Scott. 1986. MSTATC. Crop and Soil Science Department, Michigan State University, Michigan, USA.

Gallandt, E.R., M. Liebman, and D.R. Huggins. 1999. Improving soil quality: implications for weed management. Journal of Crop Production 2:95-121.

Holmes, G.J., and K.S. Mayberry. 1996. Adverse effects of sudan grass residue on lettuce stand establishment. p. 4-5. Imperial County, CA Ag Briefs, University of California Cooperative Extension, USA.

Jamil, M., Z.A. Cheema, M.N. Mushtaq, M. Farooq, and M.A. Cheema. 2009. Alternative control of wild oat and canary grass in wheat fields by allelopathic plant water extracts. Agronomy for Sustainable Development 29:475-482.
Khanh, T.D., M.I. Chung, T.D. Xuan, and S. Twata. 2005. The exploitation of crop allelopathy in sustainable agricultural production. Journal of Agronomy and Crop Science 191:172-184.

Kruidhof, H.M., E.R. Gallandt, E.R. Harmaoto, and L. Bastiaans. 2010. Selective weed suppression by cover crop residues. Effect of seed mass and time of species' selectivity. Weed Research 51:177-186.

Leishman, M.R., I.J. Wright, A.T. Moles, and M. Westoby. 2000 The evolutionary ecology of seed size. p. 31-57. In M. Fenner (ed.) Seeds: The ecology of regeneration in plant communities. CAB International, Wallingford, UK.

Liebman, M., and D.N. Sundberg. 2006 Seed mass affects the susceptibility of weed and crop species to phytotoxins extracted from red clover shoots. Weed Science 54:340-345.

Macias, F.A., T. Ascension, J.L.G. Galindo, M. Rosa, A.J. Varela, and J.M.G. Molinillo. 2002. Bioactive terpinoids from sunflower leaves cv. Peredovick. Phytochemistry 61:687-692.

Matloob, A., A. Khaliq, M. Farooq, and Z. A. Cheema. 2010. Quantification of allelopathic potential of different crop residues for the purple nutsedge suppression. Pakistan Journal of Weed Science Research 16:1-12.

Mitchell, J., C. Summers, T. Prather, J. Stapleton, and L. Roche. 2000. Potential allelopathy of sorghum-sudan mulch. HortScience 35:442.

Nelson, C.J. 1996. Allelopathy in cropping systems. Agronomy Journal 88:991-996.

Norsworthy, N.S., L. Bradenberger, N.R. Burgos, and M. Riley. 2005. Weed suppression in Vigna unguiculata with a springseeded brassicaceae green manure. Crop Protection 24:441-447.

Petersen, J.R., F. Belz, Walker, and K. Hurle. 2001. Weed suppression by release of isothiocyanates from turnip-rape mulch. Agronomy Journal 93:37-43.

Rao, A.N., D.E. Johnson, B. Sivaparsad, J.K. Ladha, and A.M. Mortimer. 2007. Weed management in direct seeded rice. Advances in Agronomy 93:153-255.

Reddy, K.N. 2001. Effect of cereal and legume cover crop residues on weeds, yield and net return in soybean (Glycine max). Weed Technology 15:660-668.

Rice, E.L. 1984. Allelopathy. p. 309-316. $2^{\text {nd }}$ ed. Academic Press Inc., London, UK

Sadeghi, S., A. Rahnavard, and Z.Y. Ashrafi. 2010. Response of wheat (Triticum aestivum) germination and growth of seedling to allelopathic potential of sunflower (Helianthus annuus) and barley (Hordeum vulgare L.) extracts. Journal of Agricultural Technology 6:573-577.

Swain, T., and W.E. Hillis. 1959. The phenolic constituents of Prunus domestica. Journal of the Science Food and Agriculture 10:63-68.

Westoby, M., D.S. Falster, A.T. Moles, P.A. Vesk, and I.J. Wright. 2002. Plant ecological strategies: some leading dimensions of variation between species. Annual Review of Ecology and Systematics 33:125-159.

Weston, L.A. 1996. Utilization of allelopathy for weed management in agro-ecosystems. Agronomy Journal 88:860-866.

Xuan, T.D., E. Tsuzuki, S. Tawata, and T. D. Khanh. 2004. Methods to determine allelopathic potential of crop plants for weed control. Allelopathy Journal 13:149-164 